\title{
Effects of the visual-feedback-based force platform training with functional electric stimulation on the balance and prevention of falls in older adults: a randomized controlled trial
}

\author{
Zhen Li ${ }^{1,2}$, Xiu-Xia Wang ${ }^{3}$, Yan-Yi Liang ${ }^{1}$, Shao-Jun Ma ${ }^{\text {Corresp., }}{ }^{1}$, Shu-Yan Chen Corresp.., 2 , Jing Sheng ${ }^{\text {Corresp. } 1}$ \\ 1 Department of Geriatrics, Shanghai Ninth People's Hospital, Shanghai Jiao Tong University School of Medicine, Shanghai, China \\ 2 Department of Geriatrics, Xinhua Hospital Affiliated to Shanghai Jiao Tong University School of Medicine, Shanghai, China \\ 3 Department of Plastic and Reconstructive Surgery, Shanghai Ninth People's Hospital, Shanghai Jiao Tong University School of Medicine, Shanghai, China \\ Corresponding Authors: Shao-Jun Ma, Shu-Yan Chen, Jing Sheng \\ Email address: dabian2017@sina.com, chenshuyan@xinhuamed.com.cn, zhenli1991@sjtu.edu.cn
}

Background. Force platform training with functional electric stimulation aimed at improving balance may be effective in fall prevention for older adults. Aim of the study is to evaluate the effects of the visual-feedback-based force platform balance training with functional electric stimulation on balance and fall prevention in older adults. Methods. $\mathrm{A}$ single-centre, unblinded, randomized controlled trial was conducted. One hundred and twenty older adults were randomly allocated to two groups: the control group ( $n=60$, oneleg standing balance exercise, $12 \mathrm{~min} / \mathrm{d}$ ) or the intervention group $(n=60$, force platform training with functional electric stimulation, $12 \mathrm{~min} / \mathrm{d}$ ). The training was provided 15 days a month for 3 months by physical therapists. Medial-lateral and anterior-posterior maximal range of sway with eyes open and closed, the Berg Balance Scale, the Barthel Index, the Falls Efficacy scale-International were assessed at baseline and after 3-month intervention. A fall diary was kept by each participant during the 6-month follow-up. Results. On comparing the two groups, the intervention group showed significantly decreased $(p<0.01)$ medial-lateral and anterior-posterior maximal range of sway with eyes open and closed. There was significantly higher improvement in the Berg Balance Scale $(p<0.05)$, the Barthel Index $(p<0.05)$ and the Falls Efficacy Scale-International $(p<0.05)$, along with significantly lesser number of injurious fallers $(p<0.05)$, number of fallers $(p<0.05)$, and fall rates $(p<0.05)$ during the 6 months follow-up in the intervention group. Conclusion. This study showed that the visual feedback-based force platform training with functional electric stimulation improved balance and prevented falls in older adults. 
1 Effects of the visual-feedback-based force platform training with functional electric stimulation

2 on the balance and prevention of falls in older adults: a randomized controlled trial

3 Zhen Li ${ }^{1,2}$, Xiu-Xia Wang ${ }^{3}$, Yan-Yi Liang ${ }^{2}$, Shao-Jun $\mathrm{Ma}^{2}$, Shu-Yan Chen ${ }^{1}$, Jing Sheng ${ }^{2}$

$4 \quad{ }^{1}$ Department of Geriatrics, Xinhua Hospital Affiliated to Shanghai Jiao Tong University School

5 of Medicine, Shanghai, China

$6 \quad{ }^{2}$ Department of Geriatrics, Shanghai Ninth People's Hospital, Shanghai Jiao Tong University

7 School of Medicine, Shanghai, China

$8{ }^{3}$ Department of Plastic and Reconstructive Surgery, Shanghai Ninth People's Hospital, Shanghai

9 Jiao Tong University School of Medicine, Shanghai, China

10 Corresponding author:

11 Jing Sheng

12 E-mail: zhenli1991@sjtu.edu.cn 


\section{Abstract}

14 Background. Force platform training with functional electric stimulation aimed at improving 15 balance may be effective in fall prevention for older adults. Aim of the study is to evaluate the 16 effects of the visual-feedback-based force platform balance training with functional electric 17 stimulation on balance and fall prevention in older adults.

18 Methods. A single-centre, unblinded, randomized controlled trial was conducted. One hundred 19 and twenty older adults were randomly allocated to two groups: the control group ( $\mathrm{n}=60$, one-leg 20 standing balance exercise, $12 \mathrm{~min} / \mathrm{d})$ or the intervention group $(\mathrm{n}=60$, force platform training with

21 functional electric stimulation, $12 \mathrm{~min} / \mathrm{d}$ ). The training was provided 15 days a month for 3

22 months by physical therapists. Medial-lateral and anterior-posterior maximal range of sway with

23 eyes open and closed, the Berg Balance Scale, the Barthel Index, the Falls Efficacy scale-

24 International were assessed at baseline and after 3-month intervention. A fall diary was kept by 25 each participant during the 6-month follow-up.

26 Results. On comparing the two groups, the intervention group showed significantly decreased $27(\mathrm{p}<0.01)$ medial-lateral and anterior-posterior maximal range of sway with eyes open and 28 closed. There was significantly higher improvement in the Berg Balance Scale $(p<0.05)$, the 29 Barthel Index $(\mathrm{p}<0.05)$ and the Falls Efficacy Scale-International $(\mathrm{p}<0.05)$, along with 30 significantly lesser number of injurious fallers $(\mathrm{p}<0.05)$, number of fallers $(\mathrm{p}<0.05)$, and fall rates $31(\mathrm{p}<0.05)$ during the 6 months follow-up in the intervention group.

32 Conclusion. This study showed that the visual feedback-based force platform training with 33 functional electric stimulation improved balance and prevented falls in older adults. 


\section{Introduction}

35 A fall is defined as an event that results in a person coming to rest inadvertently on the ground,

36 or floor, or other lower level [1]. Approximately 30\% of community-dwelling adults aged 65

37 years and older fall at least once a year [2]. The consequences of falls may be severe, leading to

38 fractures, institutionalization, loss of functional independence, disability, fear of falling,

39 depression and social isolation [3]. Therefore, prevention of falls among older adults is a crucial

40 public health challenge.

41 Balance deficits are one of the known risk factors for falls in older adults [4]. Previous meta-

42 analyses have shown that multifactorial intervention involving balance training could reduce

43 falls $[5,6]$. Some traditional balance exercises like Tai Chi as a single intervention has also

44 shown reduced risk of falls in community-dwelling older adults [7]. Recently, there is a popular

45 balance training method by instructing participants to stand on a force platform and minimizing

46 the center of pressure (COP) movement [8]. Force platform provides a method to quantify and

47 train the ability of maintaining standing position in different circumstances [9]. Older adults tend

48 to rely on exteroceptive information and prioritize the vision on postural control. Visual feedback

49 balance training can enhance sensorimotor integration by a recalibration of the sensory systems

50 contributing to balance control [10]. Force platform balance training combined with visual

51 feedback seems to effect to improve balance control and reduce postural sway in older adults

$52[11]$.

53 The ability of lower extremity muscle to generate adequate force is a fundamental component of

54 maintaining balance and a decrease in muscle strength with aging has been well documented

55 [12]. Functional electrical stimulation can increase muscle strength, induce changes in muscle

56 fiber composition and capillary system structure, prevent muscle atrophy due to the prolonged 
57 immobilization, decreases pain, and increase functional fitness [13]. Functional electrical

58 stimulation has been used to improve balance in adults with stroke and was recently shown some

59 effects on balance in older adults [14].

60 However, the effects of the visual-feedback-based force platform balance training with

61 functional electric stimulation on the prevention of falls in older adults were unknown. Therefore,

62 the purpose of this study was to evaluate the effects of the visual-feedback-based force platform

63 balance training with functional electric stimulation on the balance and prevention of falls in

64 older adults.

65 Methods

66 Participants

67 This study was conducted at a department of geriatrics in China. Recruitment started in

68 December 2015 and follow-up was completed in January 2017. Inclusion criteria were (1) age 60

69 and older, (2) ability to walk with or without an assistive device for a minimum of 20 meters, (3)

70 ability to see visual feedback from a computer screen, and (4) ability to follow instructions for

71 testing and training (Mini-Mental State Examination, MMSE, $>23$ points). Subjects were

72 excluded for any of following reasons: (1) neurological disorders, (2) severe psychiatric

73 disorders (Hamilton Depression Scale, HAMD, > 21 point, Hamilton Anxiety Scale, HAMA, >

7420 point), (3) severe musculoskeletal impairment, (4) terminal cardiovascular problems, and (5)

75 participation in other balance training.

76 All included participants signed informed consent and they were randomly assigned to the

77 intervention group (IG) or the control group (CG) using computer-generated random numbers.

78 The sequence was concealed until group assignment and baseline measurement was completed.

79 A person unrelated to the study performed the randomization procedure. This study was 
80

81

82

83

84

85

86

87

88

89

90

91

92

93

94

95

96

97

approved by the Chinese Ethics Committee of Registering Clinical Trials (ChiECRCT-20150041)

and was registered at www.Chictr.org (identified: ChiCTR-IOR-16007691). The study design

and flow of participants are shown in Figure 1.

\section{Intervention}

The IG was trained by the computerized force platform with visual feedback (Balance-A, NCC, Shanghai, China) and the functional electrical stimulation with biofeedback (MyoNet-COW, NCC, Shanghai, China).

Participants were instructed to stand barefoot on the force platform in marked foot position (10 $\mathrm{cm}$ between heel centers, with an angle of 150 between the long axes of the feet), keeping their arms by their sides. The force platform was connected to a computer and a monitor. The monitor was placed approximately $1 \mathrm{~m}$ in front of the participant at eye level. Participants could receive real-time visual feedback of their center of pressure (COP) on the monitor. First, the feedback of their anterior-posterior COP was presented as a fluctuating horizontal line that moved upwards as the COP moved forwards and downwards as the COP moved backwards. A horizontal midline indicating the correct anterior-posterior COP and two horizontal lines indicating 5\% skewing of the correct COP were placed on the screen. Participants were told that the feedback line represented the anterior-posterior position of their body and were instructed to keep the line as close to the midline as possible and not exceed the upper and lower boundary lines for 3 min. Then, the feedback of their medial-lateral COP was presented as a fluctuating vertical line. A vertical midline indicating the correct medial-lateral COP and two vertical lines indicating 5\% skewing of the correct COP were placed on the screen. Participants were asked to keep the line as close to the midline as possible and to stay in the two vertical boundary lines for $3 \mathrm{~min}$. 
102 After the force platform training, participants were instructed to performed the functional

103 electrical stimulation training in sitting position, with the ankle, knee, and hip joints at $90^{\circ}$. First,

104 Self-adhesive $5 \times 5 \mathrm{~cm}$ electrodes were placed on each leg with the anode with membrane-

105 depolarizing properties positioned over the proximal part of the tibialis anterior muscle about 5

$106 \mathrm{~cm}$ below the head of fibula. The cathode was placed on the distal part of the tibialis anterior

107 muscle about $5 \mathrm{~cm}$ above the lateral external malleolus of fibula. The starting stimulation

108 intensity was adjusted to elicit maximal contraction without inducing discomfort. Participants

109 were asked to do the ankle dorsiflexion and received electrical stimulation by the device for 3

$110 \min (10 \mathrm{~s}$ on and 10s off, rectangular-wave pulsed currents, $50 \mathrm{~Hz}$ ). Then, the anode was placed

111 along the middorsal line of shank, over both medial and lateral gastrocnemii. The cathode was

112 attached to the superficial aspect of soleus muscle $(5 \mathrm{~cm}$ in distance from where the two heads of 113 gastrocnemii join the Achilles tendon). Participants were received electrical stimulation when

114 they did the ankle plantarflexion in $3 \mathrm{~min}$.

115 In addition, both the IG and the CG performed the one-leg standing balance exercise for $12 \mathrm{~min}$

116 per day. The detailed description of one-leg standing balance exercise can be found in a previous

117 article [15]. Participants were not allowed to use a walker or cane during the training. The force

118 platform training with functional electric stimulation and the one-leg standing exercise were

119 provided 15 days a month for 3 months by two different specified physical therapists.

120 Outcome measures

121 Primary outcome: Fall rates during 6 months follow-up. Participants using daily fall diaries

122 recorded the number of falls from the day training started until the follow-up ended. The research

123 staff contacted each participant monthly to maximize compliance with the fall diaries. The fall

124 rates are the total number of falls per person in 6 months. 
125 Secondary outcomes: The number of injurious fallers and the number of fallers in 6 months

126 follow-up. Falls were classified as injurious if they resulted in a physical injury including

127 bruising, laceration, dislocation, fracture, loss of consciousness, or if the patient reported

128 persistent pain [16].

129 COP-based balance parameters were measured using the force platform. Participants were asked

130 to stand still on the force platform for 30 seconds with their eyes open, then they were asked to

131 close their eyes for 30 seconds. The following parameters were calculated: maximum range of

132 sway in the medial-lateral and anterior-posterior directions. The maximum range of sway was

133 calculated from the distance between the maximum and minimum COP displacement for each

134 direction. The reliability and validity of COP-based parameters have been tested among older

135 adults [17].

136 The Berg Balance Scale (BBS) was used as a functional balance measure. It consists of 14 items

137 that are scored on a scale of 0 to 4 and is reliable and valid in elderly people. The maximum

138 score is 56, and people with scores below 45 show a risk of falling [18]. The Falls Efficacy

139 Scale-International (FES-I) was a widely accepted tool for accessing the fear of falling. It

140 consists of 16 items that are scored on a scale of 0 to 4 and the higher scores indicate high

141 concern about falling [19]. The ability to perform activities of daily living was assessed using the

142 Barthel Index (BI), which is a valid and reliable tool. The maximum score is 100 and the higher

143 scores represent greater independence [20]. The COP-based parameters, BBS, FES-I, and BI

144 were tested by a therapist initially and after 3 months of balance training. The therapist was

145 blinded to the information about the groups to which the participants belonged.

146 The sample size estimate was based on extrapolations from our pilot studies and other related

147 promising pilot work [21, 22]. Power calculations indicated that a sample size of 120 (60 in each 
148 group) would have $80 \%$ power to detect a between-group difference of $20 \%$ in the fall rates after

149 intervention with a significance level of 0.05 and a dropout rate of $10 \%$.

\section{Data analysis}

151 Independent-sample t-tests and Chi-square tests were used for baseline comparisons between the

152 IG and CG. Chi-square tests were used to compare sex distributions and fallers in the year before

153 the intervention between the two groups. The group differences in outcome measures were

154 analyzed by two-factor analysis of variance. The between-subject factor was the treatment and

155 the within-subject factor was time. Post hoc Bonferroni tests were performed to compare post-

156 training and baseline measures in each group of patients. Fall rates and the proportion of patients

157 who had falls and injurious falls were compared by Chi-square tests during 6-month follow-up.

158 Data was analyzed using SPSS (version 21, IBM, New York, USA) and considered significant at $159 \mathrm{p}<0.05$

\section{Results}

161 A flowchart outlining study participation is shown in Figure 1. A total of 143 inpatients were 162 assessed for eligibility and 120 underwent randomization (60 in each group). No patients were 163 dropped out and no training-related adverse events occurred.

164 The baseline characteristics of the participants are summarized in Table 1. There were no 165 significant differences between the two groups in terms of age, sex, BMI, blood pressure, 166 number of medications, fallers in the previous year, MMSE, HAMD, and HAMA (p>0.05).

167 The results of baseline and post-training balance assessment are given in Table 2. No significant 168 difference was found between the two groups at baseline of BI, BBS, FES-I, and COP-based 169 parameters $(\mathrm{p}>0.05)$. 
170 The BI score and BBS score were both increased in the IG (from $93.1 \pm 2.6$ to $96.3 \pm 3.2$, from

$17144.5 \pm 5.1$ to $50.1 \pm 3.1$ ) and CG (from $93.9 \pm 2.1$ to $95.1 \pm 2.7$, from $45.2 \pm 4.7$ to $48.7 \pm 3.7$ ) after

172 training and the BBS scores in both groups were increased more than 45 . There were significant

173 differences between two groups ( $\mathrm{p}<0.05$ ). The FES-I score in the IG (from $31.4 \pm 8.2$ to $22.9 \pm 7.5$ )

174 and in the $\mathrm{CG}$ (from $32.1 \pm 8.6$ to $26.8 \pm 8.1$ ) also reduced and there were significant differences

175 between two groups ( $\mathrm{p}<0.05)$. The FES-I score in the IG $<23$ indicated low concern about 176 falling.

177 The maximal range of sway in the medial-lateral and anterior-posterior directions were reduced

178 in both the groups with eyes open and eyes closed conditions at the end of the 3 months training.

179 The medial-lateral maximal range of sway reduced from $3.81 \pm 1.05$ to $2.14 \pm 0.34$ and the

180 anterior-posterior maximal range of sway reduced from $2.63 \pm 0.52$ to $1.25 \pm 0.65$ in eyes open in

181 the IG. In the CG they were reduced from $3.57 \pm 0.56$ to $2.74 \pm 0.38$ and from $2.45 \pm 0.75$ to

$1821.85 \pm 0.15$. The medial-lateral maximal range of sway reduced from $1.86 \pm 0.79$ to $0.83 \pm 0.25$ and

183 the anterior-posterior maximal range of sway reduced from $2.49 \pm 0.87$ to $1.86 \pm 0.54$ in eyes

184 closed in the IG. In the CG they were reduced from $1.56 \pm 0.94$ to $1.03 \pm 0.47$ and from $2.54 \pm 0.61$

185 to $2.16 \pm 0.73$. There was significant difference between two groups $(\mathrm{p}<0.01)$.

186 Table 3 shows the number of injurious fallers, the number of fallers, and fall rates in 6 months

187 follow-up. It was found that the number of injurious fallers during the 6 months follow-up in the

188 IG (5) was less than the CG (13) and the difference between the two groups was statistically

189 significant $(\mathrm{p}<0.05)$. The number of fallers in the IG (8) was also less than the CG (17) during

190 the 6 months follow-up and there were significant differences between two groups $(p<0.05)$. The

191 fall rates in IG were $33.3 \%$ and in CG were 53.3\% during the 6 months follow-up. There were

192 significant differences between two groups $(\mathrm{p}<0.05)$. 


\section{Discussion}

194 The results of this randomized, controlled study showed that the visual-feedback-based force 195 platform training with functional electric stimulation can significantly improve balance and 196 prevent falls in older adults. The results also showed a positive effect of the training on activities 197 of daily living and the reduction of falling fear. To the best of our knowledge, this is the first 198 study using the visual-feedback-based force platform training with functional electric stimulation 199 to test the effects on the balance and prevention of falls in older adults.

200 The maximal range of sway in the medial-lateral and anterior-posterior directions were reduced 201 significantly in the IG indicated that participants could control their center of pressure more 202 stably and accurately. This meant these participants improved the ability to maintain the standing 203 position in static circumstances and the ability to maintain standing while experiencing internally 204 produced perturbations associated with movements of their extremities [23]. The learning of 205 balance skills was facilitated by the use of visual feedback. Visual information on how the center 206 of pressure is situated and on how it moves during different tasks serves as a tool to improve the 207 volitional postural control. This result was consistent with the motor learning theory that 208 emphasized the role of feedback in the learning of motor skills [24]. The results of the BBS 209 showed improvement in the performance of functional balance after training. These indicated 210 that the participants improved their ability of balance when they did some real-life meaningful 211 activities such as transfers and stair climbing after training. These meant that the positive effect

212 of training on balance was founded in both functional balance measures as well as laboratory

213 measures. Some previous studies also used other scales to test the effect of the visual-feedback214 based balance training on balance. For example, Schwenk et al. [25] tested thirty-three older 
215 adults using visual feedback technique and founded the Timed-up-and-go improved significantly

216 in the IG.

217 This study evaluated effect of training not only on balance but also on the ability to perform the

218 activities of daily living. The results of the BI showed that the IG significantly improved the

219 ability to perform activities of daily living after training. This means they can perform everyday

220 actions more safely and independently. Srivastava et al. [9] also reported that the BI scores were

221 improved in forty-five stroke survivors after four weeks visual-feedback-based force platform

222 training. Meanwhile, the results of the FES-I showed the less concerned about falls in the IG

223 compared with the CG after training. The consequences of fear of falling include falling,

224 restriction or avoidance of daily activities, loss of independence, reduction in social activity,

225 depression and a reduction in quality of life [26]. The reduction in fear of falling improved not

226 only the confidence regarding falls but also the confidence in social activities. This indicated that

227 this training might have positive effect on life quality of older adults.

228 It should be noted the BBS score for the IG group was raised to a level that have less risk to fall

229 as previously mentioned in the Methods section [18]. The FES-I score in the IG also reached a

230 level that has low concern about falling after three months training [19]. This study found that

231 the fallers and fall rates were both decreased significantly in the IG during six months follow-up.

232 This indicated the visual-feedback-based force platform training with functional electric

233 stimulation could prevent falls in older adults. Previous studies have tested the effect of visual-

234 feedback-based force platform training as a single intervention for fall prevention in older adults.

235 Although these studies showed that the force platform training could improve balance, they

236 found that the force platform training singly might have not significant effect on prevention of

237 falls. For example, Wolf et al. [27] found no significant difference between training group and 
238 education group in the rate of falls after 15 weeks training and 4-month follow-up in community-

239 dwelling older adults. Sihvonen et al. [28] found no significant difference in number of fallers

240 between two groups after 4 weeks training and 1-year follow-up in 27 older women living in

241 residential care. They only found the recurrent falls were significantly decreased in the IG

242 compared to the CG. This maybe because visual-feedback-based force platform training

243 emphasizes more on the improvement of the organization of sensory information related to

244 postural control and has no significant effects on ankle muscles function [29]. But humans need

245 to generate appropriate torques at the ankle joint to control body sway while standing upright and

246 the decrease in the maximal strength of ankle muscles are considered a main cause of postural

247 instability [30]. In this study, the tibialis anterior muscle and triceps surae muscle were trained

248 by the functional electrical stimulation. It has been proved the functional electrical stimulation

249 can increase muscle function of lower limbs in older adults [31]. A meta-analysis included 24

250 trials showed that electrical stimulation improved the functional motor ability and normality of

251 movement in patients with stroke [32]. In addition, this study used randomization and set strict

252 exclusion criteria to minimize other risk factors' affection on falls, for example, HAMD and

253 HAMA showed no significant difference between the two groups at the baseline. Besides, the IG

254 in this study was given a total of $13.5 \mathrm{~h}$ training which was more than twice the training duration

255 of previous studies [28]. So the visual-feedback-based force platform training with functional

256 electric stimulation showed the positive effects on the prevention of falls in older adults in this

257 study. Furthermore, the injurious fallers were also decrease significantly in the IG during six

258 months follow-up. The reasons for reduction of the injurious fallers were not only the decreased

259 fallers number but also the improved ability to restore balance in response to obstacles and

260 sudden perturbations in the walking as the improved muscle function of low limbs [32]. 
261 There were limitations in the present study. First of all, a randomized controlled trial with a

262 double-blind study design was not possible because of the nature of the intervention. So the

263 study designed some methods to reduce the bias. For instance, the different exercises were

264 conducted by different physical therapists and data were collected by a specified person who was

265 blinded to the grouping condition. Secondly,

266 This study did not use other functional balance measures (such as Timed Up-and-Go) and muscle

267 strength measures. Meanwhile, Virtual Reality (VR) as a novel technology can provide visual,

268 vestibular, and somatosensory feedback [33]. It can also provide a mimic environment that the

269 participant thinks real and environment factor is an independent risk factor of falls [34,35]. So

270 future study should add VR and more measures into the training to get more enhanced effects

271 and evidences. Finally, this study did not recruit some frail older adults because of the strict

272 inclusion and exclusion criteria. So future study needed to evaluate the effect of the training on

273 the frail older adults.

274 Conclusions

275 This study showed that the visual-feedback-based force platform training with functional electric

276 stimulation could improve balance and activities of daily living in older adults, thereby

277 preventing falls. The visual-feedback-based force platform training with functional electric

278 stimulation was feasible, with good adherence and few adverse events. Future larger study is

279 needed to test the effects of the visual-feedback-based force platform training with functional

280 electric stimulation.

281 References

282 1. Hill AM, McPhail SM, Waldron N, Etherton-Beer C, Ingram K, Flicker L, Bulsara M, 283 Haines TP. 2015. Fall rates in hospital rehabilitation units after individualised patient and 
284 staff education programmes: a pragmatic, stepped-wedge, cluster-randomised controlled 285 trial. Lancet. 385(9987):2592-9.

286 2. Hirase T, Inokuchi S, Matsusaka N, Okita M. 2015. Effects of a balance training program 287 using a foam rubber pad in community-based older adults: a randomized controlled trial. 288 J Geriatr Phys Ther. 38(2):62-70.

289 3. Abreu HC, Reiners AA, Azevedo RC, Silva AM, Abreu D, Oliveira A. 2015. Incidence 290 and predicting factors of falls of older inpatients. Rev Saude publica. 49:37.

291 4. Schmid AA, Van Puymbroeck M, Koceja DM. 2010. Effect of a 12-week yoga 292 intervention on fear of falling and balance in older adults: a pilot study. Arch Phys Med $293 \quad$ Rehabil. 91:576-83

294 5. Cameron ID, Gillespie LD, Robertson MC, Murray GR, Hill KD, Cumming RG, Kerse 295 N. 2012. Interventions for preventing falls in older people in care facilities and hospitals. 296 Cochrane Database Syst Rev. 12:CD005465.

297 6. Kannus P, Sievanen H, Palvanen M, Järvinen T, Parkkari J. 2005. Prevention of falls and 298 consequent injuries in elderly people. Lancet. 366(9500):1885-93.

299 7. Gillespie LD, Robertson MC, Gillespie WJ, Sherrington C, Gates S, Clemson LM, Lamb 300 SE. 2012. Interventions for preventing falls in older people living in the community.

$301 \quad$ Cochrane Database Syst Rev. 9:CD007146.

302 8. Dos Anjos F, Lemos T, Imbiriba LA. 2016. Does the type of visual feedback information 303 change the control of standing balance? Eur J Appl Physiol. 116:1771-9

9. Srivastava A, Taly AB, Gupta A, Kumar S, Murali T. 2009. Post-stroke balance training: 305 Role of force platform with visual feedback technique. J Neurol Sci. 287(1-2):89-93. 
306 10. Hatzitaki V, Amiridis IG, Nikodelis T, Spiliopoulou S. 2009. Direction-induced effects of 307 visually guided weight-shifting training on standing balance in the elderly. Gerontology. $55: 145-52$

309 11. Lakhani B, Mansfield A. 2015. Visual feedback of the centre of gravity to optimize standing balance. Gait Posture. 41:499-503.

311 12. Moreland JD, Richardson JA, Goldsmith CH, Clase CM. 2004. Muscle weakness and falls in older adults: a systematic review and meta-analysis. J Am Geriatr Soc. 52:1121-9.

313 13. Tok F1, Aydemir K, Peker F, Safaz I, Taşkaynatan MA, Ozgül A. 2011. The effects of 314 electrical stimulation combined with continuous passive motion versus isometric exercise on symptoms, functional capacity, quality of life and balance in knee osteoarthritis: randomized clinical trial. Rheumatol Int. 31(2):177-81

317 14. Mignardot JB, Deschamps T, Le Goff CG, Roumier FX, Duclay J, Martin A, Sixt M, Pousson M, Cornu C. 2015. Neuromuscular electrical stimulation leads to physiological gains enhancing postural balance in the pre-frail elderly. Physiol Rep. 3(7):e12471

320 15. Sakamoto K, Nakamura T, Hagino H, Endo N, Mori S, Muto Y, Harada A, Nakano T, 321 Itoi E, Yoshimura M, Norimatsu H, Yamamoto H, Ochi T; Committee on Osteoporosis of The Japanese Orthopaedic Association. 2006. Effects of unipedal standing balance exercise on the prevention of falls and hip fracture among clinically defined high-risk elderly individuals: a randomized controlled trial. J Orthop Sci. 11(5):467-72.

16. Haines TP, Hill AM, Hill KD, McPhail S, Oliver D, Brauer S, Hoffmann T, Beer C. 2011. Patient education to prevent falls among older hospital inpatients: a randomized controlled trial. Arch Intern Med. 171(6):516-24. 
328 17. Li Z, Liang YY, Wang L, Sheng J, Ma SJ. 2016. Reliability and validity of center of 329 pressure measures for balance assessment in older adults. J Phys Ther Sci. 28(4):1364-7.

330 18. Nick N, Petramfar P, Ghodsbin F, Keshavarzi S, Jahanbin I. 2016. The Effect of Yoga on 331 Balance and Fear of Falling in Older Adults. PM R. 8(2):145-51.

332 19. Delbaere K, Close JC, Mikolaizak AS, Sachdev PS, Brodaty H, Lord SR. 2010. The Falls Efficacy Scale International (FES-I). A comprehensive longitudinal validation study. Age Ageing. 39(2):210-6.

20. Ali TF, Warkentin LM, Gazala S, Wagg AS, Padwal RS, Khadaroo RG; Acute Care and Emergency Surgery (ACES) Group; Acute Care and Emergency Surgery ACES Group. 2015. Self-Reported Outcomes in Individuals Aged 65 and Older Admitted for Treatment to an Acute Care Surgical Service: A 6-Month Prospective Cohort Study. J Am Geriatr Soc. 63(11):2388-94.

21. Li Z, Wang HM, Sheng J, Ma SJ. 2016. Effectiveness of force platform balance training with visual feedback in aged Chinese: a pilot study. Int J Clin Exp Med. 9(2):4163-7

342 22. Mirelman A, Rochester L, Reelick M, Nieuwhof F, Pelosin E, Abbruzzese G, Dockx K, 343 Nieuwboer A, Hausdorff JM. 2013. V-TIME: a treadmill training program augmented by 344 virtual reality to decrease fall risk in older adults: study design of a randomized $345 \quad$ controlled trial. BMC Neurol. 13:15.

346 23. Barclay-Goddard R, Stevenson T, Poluha W, Moffatt ME, Taback SP. 2004. Force platform feedback for standing balance training after stroke. Cochrane Database Syst Rev. 4:CD004129. 
349 24. Sihvonen SE, Sipila S, Era PA. 2004. Changes in postural balance in frail elderly women 350 during a 4-week visual feedback training: a randomized controlled trial. Gerontology. $351 \quad 50: 87-95$.

352 25. Schwenk M, Grewal GS, Honarvar B, Schwenk S, Mohler J, Khalsa DS, Najafi B. 2014. 353 Interactive balance training integrating sensor-based visual feedback of movement 354 performance: a pilot study in older adults. J Neuroeng Rehabil. 11:164

355 26. Kendrick D, Kumar A, Carpenter H, Zijlstra GA, Skelton DA, Cook JR, Stevens Z, 356 Belcher CM, Haworth D, Gawler SJ, Gage H, Masud T, Bowling A, Pearl M, Morris 357 RW, Iliffe S, Delbaere K. 2014. Exercise for reducing fear of falling in older people 358 living in the community. Cochrane Database Syst Rev. 11:CD009848

359 27. Wolf SL, Barnhart HX, Kutner NG, McNeely E, Coogler C, Xu T; Atlanta FICSIT 360 Group. 2003. Selected as the best paper in the 1990s: Reducing frailty and falls in older persons: an investigation of tai chi and computerized balance training. J Am Geriatr Soc. 51(12):1794-1803.

363 28. Sihvonen S, Sipila S, Taskinen S, Era P. 2004. Fall incidence in frail older women after individualized visual feedback-based balance training. Gerontology. 50(6):411-6.

29. Hagedorn DK, Holm E. 2010. Effects of traditional physical training and visual computer feedback training in frail elderly patients. A randomized intervention study. Eur J Phys Rehabil Med. 46:159-68.

30. Cattagni T, Scaglioni G, Laroche D, Van Hoecke J, Gremeaux V, Martin A. 2014. Ankle muscle strength discriminates fallers from non-fallers. Front Aging Neurosci. 6:336. 
370 31. Maggioni MA, Cè E, Rampichini S, Ferrario M, Giordano G, Veicsteinas A, Merati G.

3712010 . Electrical stimulation versus kinesitherapy in improving functional fitness in older

372 women: A randomized controlled trial. Arch Gerontol Geriatr. 50(3):e19-25

373 32. Pomeroy VM, King L, Pollock A, Baily-Hallam A, Langhorne P. 2006.

374 Electrostimulation for promoting recovery of movement or functional ability after stroke.

375 Cochrane Database Syst Rev. 2:CD003241.

376 33. Li Z, Han XG, Sheng J, Ma SJ. 2016. Virtual reality for improving balance in patients

377 after stroke: A systematic review and meta-analysis. Clin Rehabil. 30(5):432-40.

378 34. Collado-Mateo D, Dominguez-Muñoz FJ, Adsuar JC, Merellano-Navarro E, Gusi N.

379 2017. Exergames for women with fibromyalgia: a randomised controlled trial to evaluate the effects on mobility skills, balance and fear of falling. PeerJ. 5:e3211.

381 35. Fu AS, Gao KL, Tung AK, Tsang WW, Kwan MM. 2015. Effectiveness of Exergaming 382 Training in Reducing Risk and Incidence of Falls in Frail Older Adults With a History of Falls. Arch Phys Med Rehabil. 96(12):2096-102. 
Figure 1 (on next page)

Flow chart for the study 


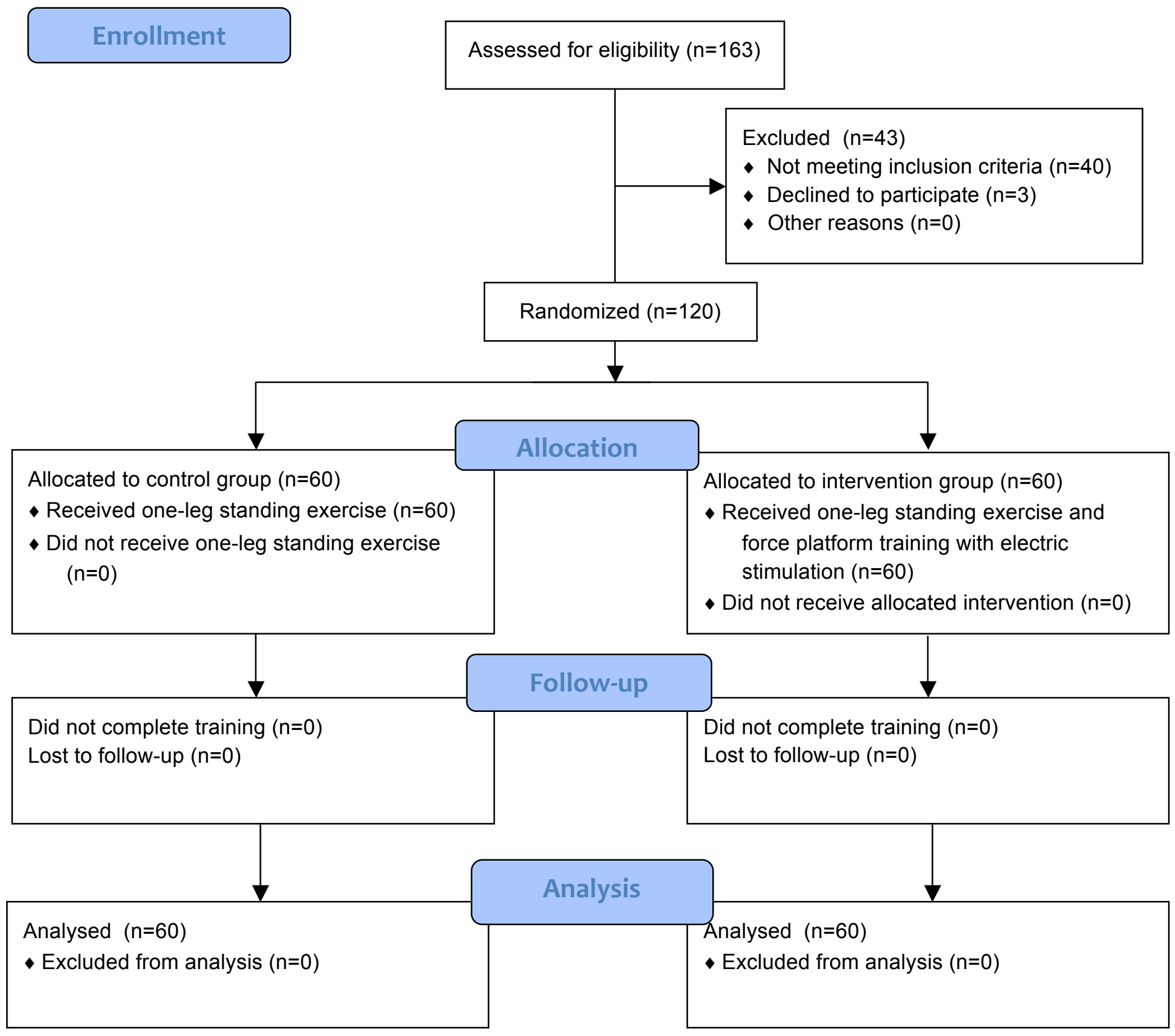


Table $\mathbf{1}$ (on next page)

Baseline characteristics of the participants 
1

\begin{tabular}{llll}
\hline Characteristic & $\begin{array}{l}\text { Intervention group } \\
(\mathrm{n}=60)\end{array}$ & $\begin{array}{l}\text { Control group } \\
(\mathrm{n}=60)\end{array}$ & P value \\
\hline Age, mean (SD), years & $69.5(6.0)$ & $70.6(5.5)$ & 0.292 \\
Females, $\mathrm{n}$ & 29 & 27 & 0.714 \\
BMI, mean (SD), kg/m² & $24.01(3.4)$ & $24.55(2.7)$ & 0.335 \\
Systolic BP, mean (SD), mmHg & $146(13.4)$ & $148(13.6)$ & 0.239 \\
Diastolic BP, mean (SD), mmHg & $83(10.1)$ & $81(8.5)$ & 0.601 \\
Number of medications, mean (SD) & $5.3(2.3)$ & $5.5(1.9)$ & 0.696 \\
Fallers in previous 12 months, $\mathrm{n}$ & 24 & 21 & 0.572 \\
MMSE, mean (SD), score & $26.9(1.6)$ & $27.3(1.7)$ & 0.342 \\
HAMA, mean (SD), score & $10.4(3.1)$ & $11.1(4.1)$ & 0.309 \\
HAMD, mean (SD), score & $13.6(4.1)$ & $12.8(3.8)$ & 0.275 \\
\hline
\end{tabular}

2 SD, standard deviation; BMI, body mass index; BP, blood pressure; MMSE, Mini-Mental State

3 Examination; HAMD, Hamilton Depression Scale; HAMA, Hamilton Anxiety Scale.

4 
Table 2 (on next page)

Effects of force platform balance training with visual feedback on balance 
2

3

\begin{tabular}{llllllll}
\hline Parameters & \multicolumn{2}{l}{ Intervention group $(\mathrm{n}=60)$} & \multicolumn{2}{l}{ Control group $(\mathrm{n}=60)$} & & \\
& Baseline & Post-training & $p^{\mathrm{a}}$ & Baseline & Post-training & $p^{\mathrm{a}}$ & $p^{\mathrm{b}}$ \\
\hline BI & $93.1(2.6)$ & $96.3(3.2)$ & $<0.001$ & $93.9(2.1)$ & $95.1(2.7)$ & 0.005 & 0.026 \\
BBS & $44.5(5.1)$ & $50.1(3.1)$ & $<0.001$ & $45.2(4.7)$ & $48.7(3.7)$ & $<0.001$ & 0.027 \\
FES-I & $31.4(8.2)$ & $22.9(7.5)$ & $<0.001$ & $32.1(8.6)$ & $26.4(8.1)$ & $<0.001$ & 0.015 \\
Eyes open & & & & & & & \\
$\mathrm{M}_{\mathrm{ML}}, \mathrm{cm}$ & $3.81(1.05)$ & $2.14(0.34)$ & $<0.001$ & $3.57(0.56)$ & $2.74(0.38)$ & $<0.001$ & 0.001 \\
$\mathrm{M}_{\mathrm{AP}}, \mathrm{cm}$ & $2.63(0.52)$ & $1.25(0.65)$ & $<0.001$ & $2.45(0.75)$ & $1.85(0.15)$ & $<0.001$ & $<0.001$ \\
Eyes closed & & & & & & & \\
$\mathrm{M}_{\mathrm{ML}}, \mathrm{cm}$ & $1.86(0.79)$ & $0.83(0.25)$ & $<0.001$ & $1.56(0.94)$ & $1.03(0.47)$ & $<0.001$ & 0.004 \\
$\mathrm{M}_{\mathrm{AP}}, \mathrm{cm}$ & $2.49(0.87)$ & $1.86(0.44)$ & $<0.001$ & $2.54(0.61)$ & $2.16(0.73)$ & 0.002 & 0.007
\end{tabular}

4

5 Data are mean (SD); ${ }^{\mathrm{a}} p$ values for within group comparisons $(p<0.05)$; ${ }^{\mathrm{b}} p$ values for treatment $\times$ time

6 interaction $(p<0.05)$; BI, Barthel Index; BBS, Berg Balance Scale; FES-I, Falls Efficacy Scale-

7 International; $\mathrm{M}_{\mathrm{ML}}$, medial-lateral maximal range of sway; $\mathrm{M}_{\mathrm{AP}}$, anterior-posterior maximal range of

8 sway.

9 


\section{Table 3 (on next page)}

Effects of force platform balance training with visual feedback on fall 
1

\begin{tabular}{llll}
\hline & IG $(\mathrm{n}=60)$ & CG $(\mathrm{n}=60)$ & P value \\
\hline Injurious Fallers, $\mathrm{n}$ & 5 & 13 & 0.041 \\
Fallers, $\mathrm{n}$ & 8 & 17 & 0.043 \\
Fall Rates, \% & 33.3 & 53.3 & 0.027
\end{tabular}

2 P values are given for difference between groups; IG, intervention group; CG, control group. 